\section{Pareto Region Characterization for Rate Control in MIMO Interference Systems and Nash Bargaining}

\author{
Zengmao Chen, Sergiy A. Vorobyov, Senior Member, IEEE, \\ Cheng-Xiang Wang, Senior Member, IEEE, and \\ John Thompson, Member, IEEE
}

\begin{abstract}
In this note, the problem of rate control in multiuser multiple-input multiple-output (MIMO) interference systems is formulated as a multicriteria optimization (MCO) problem. The Pareto rate region of the MCO problem is first characterized by giving a sufficient condition for the convexity of the Pareto rate region. Second, various rate region convexification approaches including a multistage interference cancellation and a full projection (FP)-based interference avoidance scheme are analyzed. An achievable rate region based on FP is also given for MIMO interference systems. Third, Nash bargaining (NB) is applied to transform the MCO problem into a single-objective problem. The characteristics of the NB over MIMO interference systems such as the uniqueness and optimality of different type NB solutions are investigated. A method to determine the optimality of FP- and time-division multiplexing-based NB solutions is presented as well. Finally, the convexity of the rate region and the existence of the FP-based NB solution for MIMO interference systems are examined numerically.
\end{abstract}

Index Terms-MIMO interference channel, multicriteria optimization (MCO), Nash bargaining, Pareto region.

\section{INTRODUCTION}

Multicriteria optimization (MCO), which is featured by the need to simultaneously optimize multiple noncommensurable or even incompatible objectives, has been widely used in many fields of engineering and sciences [1]. An MCO problem usually admits infinite number of noninferior solutions, which form a Pareto boundary and encompass a Pareto region [2]. The characteristics of the Pareto region are of particular interest in terms of shedding light on solving the corresponding MCO problem.

The rate control problem in multiuser interference systems can be formulated as an MCO problem. The convexity of the Pareto rate region of such an MCO problem is a desirable feature for interference

Manuscript received December 24, 2009; revised April 19, 2011; accepted April 13, 2012. Date of publication May 01, 2012; date of current version November 21,2012. This work was supported by the RCUK for the UK-China Science Bridges Project: R\&D on (B)4G Wireless Mobile Communications, the Scottish Funding Council for the Joint Research Institute in Signal and Image Processing between the University of Edinburgh and Heriot-Watt University, as part of the Edinburgh Research Partnership in Engineering and Mathematics (ERPem), by the Natural Science and Engineering Research Council (NSERC) of Canada, and by the Opening Project of the Key Laboratory of Cognitive Radio and Information Processing (Guilin University of Electronic Technology), Ministry of Education, China under Grant 2011KF01. Recommended by Associate Editor H. Zhang.

Z. Chen and C.-X. Wang are with the EECE, Joint Research Institute for Signal and Image Processing, School of Engineering and Physical Sciences, Heriot-Watt University, Edinburgh, EH14 4AS, U.K. (e-mail: zengmao.chen@hw.ac.uk; cheng-xiang.wang@hw.ac.uk).

S. A. Vorobyov is with the Department of Electrical and Computer Engineering, University of Alberta, Edmonton, AB, T6G 2V4, Canada (e-mail: svorobyo@ualberta.ca).

J. Thompson is with the Joint Research Institute for Signal and Image Processing, Institute for Digital Communications, University of Edinburgh, Edinburgh, EH9 3JL, U.K. (e-mail: john.thompson@ed.ac.uk).

Color versions of one or more of the figures in this technical note are available online at http://ieeexplore.ieee.org.

Digital Object Identifier 10.1109/TAC.2012.2197075 systems. Various approaches have been used to convexify a rate region $^{1}[3]$, [4]. One popular approach is based on the use of a convex hull of rate region [3]. However, its rate boundary is achievable only in terms of the average rate. Another approach is based on using orthogonal signaling schemes, e.g., time division multiplexing (TDM) and frequency division multiplexing (FDM) [4]. They significantly simplify the MCO problem, but they usually lead to smaller achievable rate regions [5]. Therefore, it is desirable to analyze the convexity of the true Pareto rate region and compare the resulting rate regions of different convexification schemes.

Recently, Nash bargaining (NB) theory for cooperative games has been widely recognized as a useful approach for solving the MCO problem for multiuser interference systems due to the fact that NB can effectively balance the fairness of individual users and the system-level performance [6]. Representative examples in the literature include [4] and [7] for single-input single-output (SISO), [3] and [5] for multipleinput single-output (MISO), and [8] for MIMO interference systems. However, little research has been done to characterize the pure strategy based NB for MIMO interference systems.

In this technical note, we address the MCO problem of rate control in multiuser MIMO interference systems. First, the Pareto rate region of this MCO problem is characterized. It is proved that the condition that interference-plus-noise covariance matrices approach identity matrix is a sufficient condition for the convexity of the rate region. Moreover, a significant implication of this fact is found and states that when interference is high, interference mitigation techniques are preferable for convexifying the rate region. Second, various rate region convexification approaches including a multistage interference cancellation (IC) and a full projection (FP)-based interference avoidance (IA) schemes are developed and analyzed. An achievable rate region based on FP precoding is also given for MIMO interference systems. Third, the MCO problem for rate control is scalarized by using NB. The characteristics of the NB over MIMO interference systems such as the uniqueness of the pure-strategy NB solution and the optimality of NB solutions resulting from different convexification approaches are studied.

The remainder of this technical note is organized as follows. The MCO problem and NB for multiuser MIMO interference systems are formulated in Section II. In Section III, the Pareto rate region is characterized and various rate region convexification approaches for MIMO interference systems are analyzed. The characteristics of pure-strategy NB solution and the optimality of different NB solutions are studied in Section IV. The convexity of the rate region and the existence of the FP-based NB solution for MIMO interference systems are exemplified in Section V via numerical studies. Finally, conclusions are drawn in Section VI.

\section{Notation}

For a matrix $\mathbf{X}, \operatorname{tr}[\mathbf{X}], \mathbf{X}^{-1}, \mathbf{X}^{H}$, and $\mathbf{X}^{\dagger}$ denote its trace, inverse, Hermitian transpose and pseudoinverse, respectively. $\mathbb{E}[\cdot]$ stands for the statistical expectation operator.

\section{Problem Formulation}

\section{A. MCO in MIMO Interference Systems}

Consider an $M$-user MIMO interference system. The transmitter and receiver for user $i$ are equipped with $N_{i}^{t}$ and $N_{i}^{r}$ antennas, respectively.

${ }^{1}$ When referring to rate region/boundary, we mean the Pareto rate region/ boundary hereafter. 
The $N_{i}^{r} \times 1$ complex baseband signal vector received by user $i$ can be written as [9]

$$
\mathbf{y}_{i}=\mathbf{H}_{i i} \mathbf{x}_{i}+\sum_{j=1, j \neq i}^{M} \mathbf{H}_{j i} \mathbf{x}_{j}+\mathbf{n}_{i}
$$

where $\mathbf{x}_{i} \in \mathbb{C}^{N_{i}^{t} \times 1}$ is the transmitted signal vector for user $i ; \mathbf{H}_{i i} \in$ $\mathbb{C}^{N_{i}^{r} \times N_{i}^{t}}$ and $\mathbf{H}_{j i} \in \mathbb{C}^{N_{i}^{r} \times N_{j}^{t}}$ are channel matrices from transmitter $i$ and transmitter $j$ to receiver $i$, respectively; we assume that $\mathbf{H}_{i i} \sim$ $\mathcal{C} \mathcal{N}\left(0, \rho_{i}\right)(i=1,2, \ldots, M)$, i.e., the elements of the channel matrices are independent and identically distributed (i.i.d.) circular symmetric complex Gaussian random variables with zero mean and variance $\rho_{i}$; we also have $\mathbf{H}_{j i} \sim \mathcal{C} \mathcal{N}\left(0, \eta_{j i}\right)(j=1,2, \ldots, M ; j \neq i)$; and $\mathbf{n}_{i} \in \mathbb{C}^{N_{i}^{r} \times 1}$ is the i.i.d. additive white Gaussian noise (AWGN) vector of user $i$ with zero mean and covariance matrix being identity matrix $\mathbb{E}\left[\mathbf{n}_{i} \mathbf{n}_{i}^{H}\right]=\mathbf{I}$. Then, $\rho_{i}$ is the normalized signal-to-noise ratio (SNR) for user $i$ and $\eta_{j i}$ is the normalized interference-to-noise ratio (INR).

The mutual information for user $i$ can be expressed as [10]

$$
I_{i}(৫)=\log _{2} \operatorname{det}\left(\mathbf{I}+\mathbf{H}_{i i} \mathbf{Q}_{i} \mathbf{H}_{i i}^{H} \mathbf{R}_{-i}^{-1}\right)
$$

where $Q \triangleq\left\{\mathbf{Q}_{1}, \ldots, \mathbf{Q}_{M}\right\}, \mathbf{Q}_{i}=\mathbb{E}\left[\mathbf{x}_{i} \mathbf{x}_{i}^{H}\right]$ is the Hermitian positive semi-definite (PSD) transmit covariance matrix of the input signal vector for user $i$, i.e., $\mathbf{Q}_{i} \succeq 0$, and

$$
\mathbf{R}_{-i}=\mathbf{I}+\sum_{j=1, j \neq i}^{M} \mathbf{H}_{j i} \mathbf{Q}_{j} \mathbf{H}_{j i}^{H}
$$

is the interference-plus-noise covariance matrix for user $i$. The transmission of each user is power limited, i.e.

$$
\operatorname{tr}\left(\mathbf{Q}_{i}\right) \leq p_{i}, \quad i=1,2, \ldots, M
$$

The rate control objective in the MIMO interference system is the multiple rates ${ }^{2}$ maximization for all users by optimizing their transmit covariance matrices under the power constraints (4). Therefore, the rate control problem can be formulated as the following $\mathrm{MCO}$ problem:

$$
\begin{array}{r}
\max _{\ell} I_{i}(\varrho) \text { for all } i=1,2, \ldots, M \\
\text { subject to } \mathbf{Q}_{i} \succeq 0, \quad i=1,2, \ldots, M \\
\operatorname{tr}\left(\mathbf{Q}_{i}\right) \leq p_{i}, \quad i=1,2, \ldots, M .
\end{array}
$$

\section{B. Scalarization of the $M C O$ Using $N B$}

In this technical note, we restrict our attention to the NB as one of the most popular approaches to scalarize the multiple objectives of the MCO problem (5) [6]. In the context of the MIMO interference game with a convex rate region, the bargaining set, i.e., the set of available strategies for user $i(i=1,2, \ldots, M)$, can be expressed as

$$
\mathscr{S}=\left\{\mathbf{Q}_{i} \mid \mathbf{Q}_{i} \succeq 0, \operatorname{tr}\left(\mathbf{Q}_{i}\right) \leq p_{i}, \text { and } I_{i}(Q)>I_{i}^{\mathrm{NE}}\right\}
$$

where $I_{i}^{\mathrm{NE}}$ is the utility of the Nash equilibrium (NE) for user $i$. NE is a steady state in competitive games for which each player can not improve its utility by unilaterally changing its own strategy[11]. The transmit covariance matrix leading to the NE can be found via iterative water filling (IWF) as [12]

$$
\mathbf{Q}_{i}^{*}=\mathbf{U}_{i}\left(\mu_{i} \mathbf{I}-\mathbf{D}_{i}^{-1}\right)^{+} \mathbf{U}_{i}^{H}, \quad i=1,2, \ldots, M
$$

${ }^{2}$ Hereafter, when referring to rate, we mean mutual information (2). where $\mathbf{U}_{i} \mathbf{D}_{i} \mathbf{U}_{i}^{H}=\mathbf{H}_{i i}^{H} \mathbf{R}_{-i}^{-1} \mathbf{H}_{i i}$ is the eigenvalue decomposition (EVD) of $\mathbf{H}_{i i}^{H} \mathbf{R}_{-i}^{-1} \mathbf{H}_{i i}$, and $\mu_{i}$ denotes the power level given by IWF.

By applying the NB principle, the MCO problem (5) can be scalarized as the following single-objective optimization problem:

$$
\begin{aligned}
\max _{\ell} \prod_{i=1}^{M}\left(I_{i}(Q)-I_{i}^{\mathrm{NE}}\right) \\
\text { subject to } \mathbf{Q}_{i} \succeq 0, \quad i=1,2, \ldots, M \\
\operatorname{tr}\left(\mathbf{Q}_{i}\right) \leq p_{i}, \quad i=1,2, \ldots, M \\
I_{i}(\varrho)>I_{i}^{\mathrm{NE}}, \quad i=1,2, \ldots, M .
\end{aligned}
$$

The NB maximizes the Nash product of the whole MIMO system while guaranteeing that the utility of each MIMO user is not less than that of the NE.

\section{RATE REGION CHARACTERIZATION AND CONVEXIFICATION}

\section{A. Convexity of Pareto Rate Region}

In the context of the MIMO interference systems, we first investigate the convexity of the true Pareto rate region using pure strategies. The true Pareto rate region is achievable in terms of instantaneous rate and it is usually larger than the orthogonal signaling-based counterpart in low interference cases [5]. We derive the following sufficient condition ensuring the convexity of the rate region.

Proposition 1: If the interference-plus-noise covariance matrices $\mathbf{R}_{-i} \rightarrow \mathbf{I}(i=1,2, \ldots, M)$, the Pareto rate region of the MIMO interference system is convex.

The detailed proof can be found in [13]. The condition that $\mathbf{R}_{-i} \rightarrow \mathbf{I}$ corresponds to the condition that INRs $\eta_{j i}(i, j=1,2, \ldots, M ; j \neq$ $i$ ) in (1) are sufficiently small. Hence, the immediate implication of Proposition 1 is that the rate region is convex when the interference is low. Then, the following remark on rate region convexification can be made.

Remark 1: Interference mitigation techniques can be used to convexify the rate region besides the use of convex hull and orthogonal signaling.

The rate region convexification schemes can be summarized as the following three categories.

- Convex hull - It stems from the time-sharing signaling of extremity points on the rate boundary, where all users agree to use one set of transmitting strategies for certain fraction of time and to use the other set of strategies during the rest of the time (see, for example [3]). Therefore, the time-sharing signaling fills the "depressions" of the rate region and make it convex. However, its rate region is only achievable in terms of average rate rather than instantaneous rate [3].

- Orthogonal signaling - In this case, users agree to split the radio resource (time or bandwidth) into several orthogonal parts and each user uses one part only. TDM and FDM are two prominent examples of orthogonal signaling. It worth noting that time sharing involves in both TDM and convex hull. TDM distinguishes itself from the convex hull in the sense that the former is pure time-sharing signaling among single-user operation points, while the latter is time sharing of extremity points as shown in Fig. 1. The orthogonal signaling significantly simplifies the MCO problem of rate control, but the associated rate loss may be large compared to the pure strategy approach [5].

- Interference mitigation - Proposition 1 suggests that reducing interference for each user can also make the rate region convex. Therefore, various interference mitigation techniques are applicable to convexify the rate region. Interference mitigation can be mainly divided into two categories : i) interference cancellation at the receiver and ii) interference avoidance at the transmitter. 


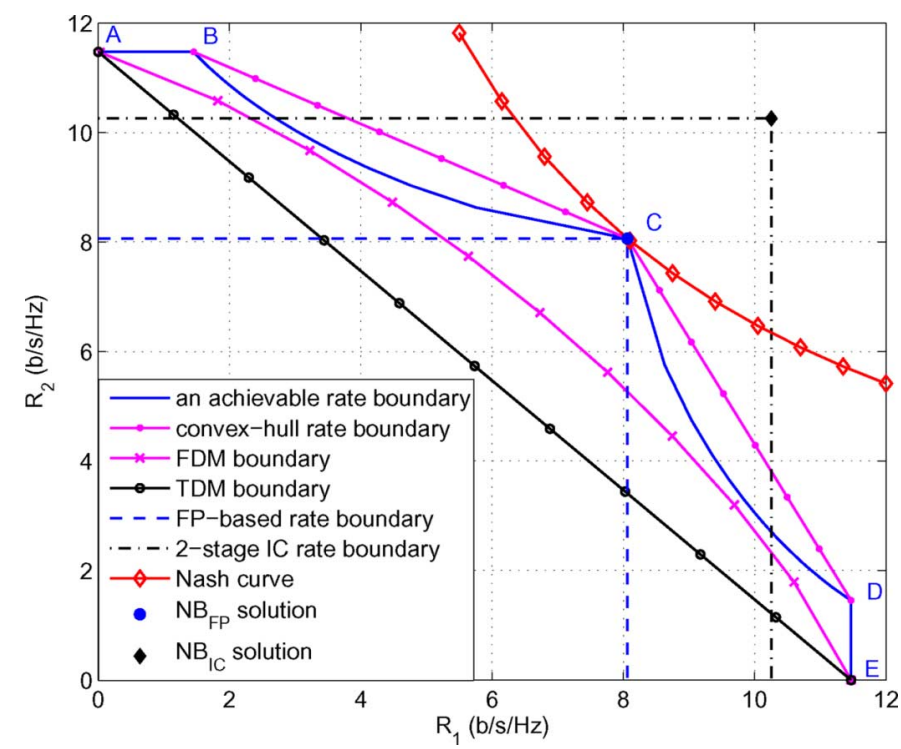

Fig. 1. Example of different signaling schemes and NB solutions of 2-user MIMO interference system.

\section{B. Convexification Using Interference Cancellation}

Various interference cancellation techniques [14] can be used for interference systems. The following comparison between orthogonal signaling and interference cancellation is of interest. Orthogonal signaling is a simple and widely used method to produce a convex rate region at the cost of rate loss. Application of interference cancellation techniques to an interference system eventually transforms a high-interference system into a low-interference system, which consequently leads to a convex rate region according to Proposition 1. Moreover, interference cancellation removes the interference perceived at the receivers, but unlike orthogonal signaling, the radio resource (time, bandwidth) available to each user for interference cancellation remains the same as for pure strategy. Therefore, the following remark about the practical significance of the rate region convexification can be made.

Remark 2: When the interference is high, interference cancellation techniques outperform orthogonal signaling techniques for convexifying a rate region in the sense that they lead to a convex rate region of a larger size.

We can obtain the immediate conclusion from Remark 2 that interference cancellation ensures the uniqueness of NB solution, meanwhile, the interference cancellation-based $\mathrm{NB}\left(\mathrm{NB}_{\mathrm{IC}}\right)$ solution has higher user rates than the orthogonal signaling based NB solution.

As a special case, we propose a multistage interference cancellation technique for a 2-user MIMO interference system. The generalization to the multiuser case is straightforward and omitted here for brevity. It is worth noting that our objective is to demonstrate the advantages of the interference cancellation over orthogonal signaling for the multicriteria optimization via NB rather than considering practically appealing interference cancellation designs.

The multistage interference cancellation works as follows. First, the receivers of users 1 and 2 decode their signals of interest and pass them to the receivers of users 2 and 1, respectively. Using (1), the decoded signals from receivers of users 1 and 2 in the first stage of interference cancellation can be written as

$$
\begin{aligned}
& \hat{\mathbf{x}}_{1}^{(1)}=\mathbf{H}_{11}^{\dagger} \mathbf{y}_{1} \\
& \hat{\mathbf{x}}_{2}^{(1)}=\mathbf{H}_{22}^{\dagger} \mathbf{y}_{2} .
\end{aligned}
$$

Second, the receivers of users 1 and 2 reconstruct the interference signals from the decoded signals and subtract them from its received signal to obtain the following signals used in the second stage of the interference cancellation procedure

$$
\begin{aligned}
\mathbf{y}_{1}^{(2)} & =\mathbf{y}_{1}-\mathbf{H}_{21} \hat{\mathbf{x}}_{2}^{(1)} \\
& =\left(\mathbf{H}_{11}-\mathbf{H}_{21} \mathbf{H}_{22}^{\dagger} \mathbf{H}_{12}\right) \mathbf{x}_{1}+\mathbf{n}_{1}-\mathbf{H}_{21} \mathbf{H}_{22}^{\dagger} \mathbf{n}_{2} \\
\mathbf{y}_{2}^{(2)} & =\mathbf{y}_{2}-\mathbf{H}_{12} \hat{\mathbf{x}}_{1}^{(1)} \\
& =\left(\mathbf{H}_{22}-\mathbf{H}_{12} \mathbf{H}_{11}^{\dagger} \mathbf{H}_{21}\right) \mathbf{x}_{2}+\mathbf{n}_{2}-\mathbf{H}_{12} \mathbf{H}_{11}^{\dagger} \mathbf{n}_{1} .
\end{aligned}
$$

The decoding, reconstruction, and subtraction procedure is repeated stage after stage and terminated after a certain stage or after a stopping tolerance criteria on the remaining error is satisfied. An example of a 2-stage interference cancellation is demonstrated in Fig. 1 for a 2-user MIMO interference system.

\section{Rate Region Convexification Using Interference Avoidance}

Interference avoidance refers to proactive approaches employed at the transmitter side to avoid interfering unintended receivers. In the context of MIMO interference systems, null space projection-based precoding is one of the commonly used interference avoidance schemes. It steers the transmission of each MIMO transmitter into the null space of interfering channel to its unintended receivers. As an example of null space projection-based precoding, FP precoding [15] is capable of completely avoiding interfering unintended receivers and transforming the MIMO interference system into an interference-free system. Therefore, it can be used to convexify the rate region of MIMO interference system.

To facilitate the FP precoding, the number of transmit antennas of each user must be larger than the total number of receive antennas of all unintended receivers, i.e.

$$
N_{i}^{t}>\sum_{j \neq i} N_{j}^{r} \quad \forall i, j=1,2, \ldots, M .
$$

For user $i$, its transmit covariance matrix using FP precoding with transmission power $p_{i}$ can be written as [15]

$$
\mathbf{Q}_{\perp i}\left(p_{i}\right)=\mathbf{U}_{\perp i}\left(\mu_{i} \mathbf{I}-\boldsymbol{\Lambda}_{\perp i}^{-1}\right)^{+} \mathbf{U}_{\perp i}^{H}
$$

where $\mu_{i}$ is the power level obtained from the water filling, $\operatorname{tr}\left(\mu_{i} \mathbf{I}-\right.$ $\left.\boldsymbol{\Lambda}_{\perp i}^{-1}\right)^{+}=p_{i}$. In (14), $\mathbf{U}_{\perp i}$ and $\boldsymbol{\Lambda}_{\perp i}$ are obtained from the EVD $\mathbf{U}_{\perp i} \Lambda_{\perp i} \mathbf{U}_{\perp i}^{H}=\left(\mathbf{I}-\mathbf{U}_{g i} \mathbf{U}_{g i}^{H}\right)^{H} \mathbf{H}_{i i}^{H} \mathbf{H}_{i i}\left(\mathbf{I}-\mathbf{U}_{g i} \mathbf{U}_{g i}^{H}\right)$, and $\mathbf{U}_{g i}$ is the matrix consisting of the first $\sum_{j \neq i} N_{j}^{r}$ columns of the unitary matrix $\mathbf{U}_{G}$ stemming from another $\operatorname{EVD} \mathbf{U}_{G} \boldsymbol{\Lambda}_{G}^{1 / 2} \mathbf{V}_{G}^{H}=\mathbf{H}_{-i}^{H}$, where

$$
\mathbf{H}_{-i}=\left[\mathbf{H}_{i 1} ; \ldots ; \mathbf{H}_{i i-1} ; \mathbf{H}_{i i+1} ; \ldots ; \mathbf{H}_{i M}\right]
$$

denotes the vertically concatenated interference channels from user $i$ to all its unintended users $j(j=1,2, \ldots, M ; j \neq i)$.

The rate region of FP precoding for a 2 -user MIMO interference system is also shown in Fig. 1. As it can be seen from this figure, the FP precoding based rate region is larger than the orthogonal signaling counterparts in the top right region. This observation leads to another remark on rate region convexification.

Remark 3: To convexify a rate region, interference avoidance has potential to outperform the orthogonal signaling when the fairness requirement to each user is imposed.

The performance of the aforementioned rate region convexification schemes is summarized in Table I, where the requirements on channel state information (CSI) and receiver ( $\mathrm{Rx}$ ) cooperation, implementation complexity, and the size of resultant rate region are compared. 
TABLE I

COMPARISON OF VARIOUS RATE REGION CONVEXIFICATION SCHEMES

\begin{tabular}{|c||c|c|c|c}
\hline & CSI & Rx cooperation & Complexity & Rate region \\
\hline \hline TDM & unrequired & unrequired & low & small \\
\hline FDM & unrequired & unrequired & low & medium \\
\hline Multistage IC & required & required & high & large \\
\hline FP-based IA & required & unrequired & medium & medium
\end{tabular}

\section{Rate Region Characterization}

In what follows, we give an achievable rate region for MIMO interference systems satisfying (13). We start with a 2-user MIMO system. Its rate boundary can be obtained by finding the rate tuple $\left(R_{1}, \max _{\mathrm{Q}}\left(I_{2}\right)\right)$. Given a rate $R_{1}$ for user 1 , the corresponding rate $R_{2}$ for user 2 on the rate boundary is the maximum rate which can be achieved by user 2 when i) there is no interference from user 1 to user 2 , i.e., user 1 adopts the FP precoding with transmit covariance matrix $\mathbf{Q}_{\perp 1}$, and ii) user 2 employs the maximum rate precoding with the following transmit covariance matrix and full-power transmission

$$
\mathbf{Q}_{\mathrm{MR}_{2}}\left(p_{2}\right)=\mathbf{U}_{\mathrm{MR}_{2}}\left(\mu_{2} \mathbf{I}-\boldsymbol{\Lambda}_{\mathrm{MR}_{2}}^{-1}\right)^{+} \mathbf{U}_{\mathrm{MR}_{2}}^{H}
$$

where $\mathbf{U}_{\mathrm{MR}_{2}}$ and $\boldsymbol{\Lambda}_{\mathrm{MR}_{2}}$ come from the EVD $\mathbf{U}_{\mathrm{MR}_{2}} \boldsymbol{\Lambda}_{\mathrm{MR}_{2}} \mathbf{U}_{\mathrm{MR}_{2}}^{H}=$ $\mathbf{H}_{22}^{H} \mathbf{H}_{22}$. Therefore, an achievable rate boundary can be obtained as follows:

- For $I_{1}\left(\mathbf{Q}_{\perp_{1}}(0), \mathbf{Q}_{\mathrm{MR}_{2}}\left(p_{2}\right)\right) \leq R_{1} \leq I_{1}\left(\mathbf{Q}_{\perp_{1}}\left(p_{1}\right)\right.$, $\left.\mathbf{Q}_{\mathrm{MR}_{2}}\left(p_{2}\right)\right),{ }^{3} R_{2}$ can achieve its largest rate. This part of rate boundary $\mathbb{R}_{1}$ can be expressed as

$$
\begin{aligned}
\mathbb{R}_{1} & : 0 \leq R_{1} \leq I_{1}\left(\mathbf{Q}_{\perp_{1}}\left(p_{1}\right), \mathbf{Q}_{\mathrm{MR}_{2}}\left(p_{2}\right)\right) \\
R_{2} & =\log _{2} \operatorname{det}\left(\mathbf{I}+\mathbf{H}_{22} \mathbf{Q}_{\mathrm{MR}_{2}}\left(p_{2}\right) \mathbf{H}_{22}^{H}\right) \\
& =\sum_{k}\left(\log _{2}\left(p_{2} \lambda_{k}^{\mathrm{MR}_{2}}\right)\right)^{+}
\end{aligned}
$$

The second equality for $R_{2}$ in (17) is obtained according to [16], $\lambda_{n}^{\mathrm{MR}_{i}}\left(i=1,2, \ldots, M ; n=1,2, \ldots, \min \left(N_{i}^{t}, N_{i}^{r}\right)\right)$ are the diagonal elements of the eigenvalue matrix $\boldsymbol{\Lambda}_{\mathrm{MR}_{i}}$, and the operator $\sum_{k}\left(x_{k}\right)^{+}$stands for the sum of $x_{k}$ with $k \in\left\{n \mid p_{i}-1 / \lambda_{n}^{\mathrm{MR}_{i}}>\right.$ $0\}$. It is easy to see that $\mathbb{R}_{1}$ (line $A B$ in Fig. 1) coincides with the boundary of the Pareto rate region.

- At point B in Fig. 1, $R_{1}$ cannot be further improved along the line of $\mathrm{AB}$ since user 1 is transmitting at full power. However, if user 2 splits some of the transmission power for FP precoding, the interference perceived at receiver 1 caused by user 2 will reduce. Consequently, $R_{1}$ can be further improved. Then, we have the second part of the achievable rate boundary

$$
\begin{aligned}
& \mathbb{R}_{2}: R_{1} \\
& =\log _{2} \operatorname{det}\left\{\mathbf{I}+\frac{\mathbf{H}_{11} \mathbf{Q}_{\perp_{1}}\left(p_{1}\right) \mathbf{H}_{11}^{H}}{\mathbf{H}_{21}\left[\tau \mathbf{Q}_{\perp_{2}}\left(p_{2}\right)+(1-\tau) \mathbf{Q}_{\mathrm{MR}_{2}}\left(p_{2}\right)\right] \mathbf{H}_{21}^{H I}}\right\} \\
& R_{2} \\
& =\log _{2} \operatorname{det}\left\{\mathbf{I}+\mathbf{H}_{22}\left[\tau \mathbf{Q}_{\perp_{2}}\left(p_{2}\right)+(1-\tau) \mathbf{Q}_{\mathrm{MR}_{2}}\left(p_{2}\right)\right] \mathbf{H}_{22}^{H}\right\} \\
& \text { with } 0 \leq \tau \leq 1 .
\end{aligned}
$$

It is worth noting that $\mathbb{R}_{2}$ is one of the achievable rate boundaries which is not necessarily the Pareto rate boundary. In Fig. 1, the curve BC depicts $\mathbb{R}_{2}$.

- Using the symmetry of the previous results, the other two parts of the achievable rate boundary $\mathbb{R}_{3}$ and $\mathbb{R}_{4}$ (curve $C D$ and line DE in Fig. 1) are symmetric to $\mathbb{R}_{2}$ and $\mathbb{R}_{1}$, respectively.

${ }^{3} I_{i}\left(\mathbf{Q}_{1}\left(p_{1}\right), \mathbf{Q}_{2}\left(p_{2}\right)\right.$ denotes the rate of user $i$ when the transmit covariance matrices $\mathbf{Q}_{1}$ and $\mathbf{Q}_{2}$ with transmission power $p_{1}$ and $p_{2}$ are adopted.
By induction, for an $n$-user MIMO interference channel satisfying (13), one of the achievable rate boundaries can be characterized by the following two types of hypersurfaces of $n-1$ dimensions.

- One user $\left(\mathrm{C}_{n}^{1}\right)$ uses full-power maximum rate precoding $\mathbf{Q}_{\mathrm{MR}_{i}}\left(p_{i}\right)(i=1,2, \ldots, n)$. All other users adopt varying-power FP precoding, i.e., $\mathbf{Q}_{\perp_{j}}\left(\tau_{j} p_{j}\right)\left(j \neq i ; \tau_{j} \in[0,1]\right)$.

- One user $\left(\mathrm{C}_{n}^{1}\right)$ uses the convex combinational precoding strategy $\tau \mathbf{Q}_{\mathrm{MR}_{i}}\left(p_{i}\right)+(1-\tau) \mathbf{Q}_{\perp_{i}}\left(p_{i}\right),(\tau \in[0,1] ; i=1,2, \ldots, n)$. One of the other users among the remaining $n-1$ users $\left(\mathrm{C}_{n-1}^{1}\right)$ adopts the full-power FP precoding $\mathbf{Q}_{\perp_{j}}\left(p_{j}\right)(j \neq i)$. All the rest users employ varying-power FP precoding $\mathbf{Q}_{\perp_{k}}\left(\tau_{k} p_{k}\right)(k \neq i ; k \neq$ $\left.j ; \tau_{k} \in[0,1]\right)$. Thus, the total number of hypersurfaces of these two types aggregate to $\mathrm{C}_{n}^{1}+\mathrm{C}_{n}^{1} \mathrm{C}_{n-1}^{1}=n^{2}$.

\section{Characterization OF DifFERENT NB SOlutions}

\section{A. Uniqueness of Pure-Strategy NB Solution}

It is straightforward to see that the optimization problem given by (8) is identical to

$$
\begin{gathered}
\max _{\mathbf{Q}} \ln \left(\prod_{i=1}^{M}\left(I_{i}(\varrho)-I_{i}^{\mathrm{NE}}\right)\right) \\
\text { subject to } \mathbf{Q}_{i} \succeq 0, \quad i=1,2, \ldots, M \\
\operatorname{tr}\left(\mathbf{Q}_{i}\right)-p_{i} \leq 0, \quad i=1,2, \ldots, M \\
I_{i}^{\mathrm{NE}}-I_{i}(\varrho)<0, \quad i=1,2, \ldots, M .
\end{gathered}
$$

Note that (19) is a convex optimization problem if and only if its objective function is concave and its constraint set is convex [17].

Proposition 2: When the interference-plus-noise covariance matrices $\mathbf{R}_{-i} \rightarrow \mathbf{I}(i=1,2, \ldots, M)$, the optimization problem (19) is a convex problem.

This proposition has been proved in the full version of this technical note [13] by showing that $\mathbf{R}_{-i}$ approaching $\mathbf{I}$ is sufficient for both the concavity of the objective function in (19) and the convexity of its constraint set.

From Proposition 2, the following corollary can be obtained.

Corollary 1: When the NE is not on the Pareto rate boundary, the condition that the interference-plus-noise covariance matrices $\mathbf{R}_{-i}(i=1,2, \ldots, M)$ approach $\mathbf{I}$, is the sufficient condition for the uniqueness of the pure-strategy NB solution of MIMO interference systems.

Proof: The interference-plus-noise covariance matrices $\mathbf{R}_{-i}(i=$ $1,2, \ldots, M)$ approaching $\mathbf{I}$ can ensure the convexity of the rate region (see Proposition 1) on which the NB is defined. Interestingly, it also guarantees the uniqueness of the NE in MIMO interference systems (see [18, Proposition 1]), i.e., it guarantees that the IWF converges. Proposition 2 states that if the interference-plus-noise covariance matrices $\mathbf{R}_{-i}$ approach $\mathbf{I}$, then (19) is a convex optimization problem, i.e., there exists at most one solution maximizing the objective function of (19) [17]. Moreover, the NE not being on the Pareto boundary is necessary for the existence of the NB solution. Applying Propositions 1 and 2 of this technical note and [18, Proposition 1], we complete the proof.

\section{B. Optimality of NB Solutions}

Various rate region convexification schemes investigated in Sections III-B and -C lead to different NB solutions. It can be observed from Fig. 1 that the TDM and multistate interference cancellation scheme have the smallest and largest rate regions, respectively. Hence, they lead to the NB solutions with the smallest and largest user rates, respectively. Moreover, the FDM-based NB ( $\mathrm{NB}_{\mathrm{FDM}}$ ) solution is optimal over the TDM-based $\mathrm{NB}\left(\mathrm{NB}_{\mathrm{TDM}}\right)$ solution due 
to the fact that the FDM-based rate region is strictly convex while the TDM-based rate region is not. Next, we analyze the optimality of $\mathrm{NB}_{\mathrm{FDM}}$ and FP procoding-based $\mathrm{NB}\left(\mathrm{NB}_{\mathrm{FP}}\right)$ solutions.

The $\mathrm{NB}_{\mathrm{FP}}$ solution for user $i$ can be expressed as

$$
\begin{aligned}
R_{i}^{\mathrm{NB}} & \\
= & \log _{2} \operatorname{det}\left(\mathbf{I}+\mathbf{H}_{i i} \mathbf{Q}_{\perp_{i}}\left(p_{i}\right) \mathbf{H}_{i i}^{H}\right) \\
= & \max _{\mathbf{Q}_{i}} \log _{2} \operatorname{det}\left\{\mathbf{I}+\mathbf{H}_{i i}\left[\mathbf{I}-\mathbf{H}_{-i}^{H}\left(\mathbf{H}_{-i} \mathbf{H}_{-i}^{H}\right)^{-1} \mathbf{H}_{-i}\right] \mathbf{Q}_{i}\right. \\
& \left.\times\left[\mathbf{I}-\mathbf{H}_{-i}^{H}\left(\mathbf{H}_{-i} \mathbf{H}_{-i}^{H}\right)^{-1} \mathbf{H}_{-i}\right]^{H} \mathbf{H}_{i i}^{H}\right\} \\
& \quad \sum_{i}^{t}-\sum_{j \neq i}{ }^{N_{i}^{r}}\left(\log _{2}\left(p_{i} \lambda_{k}^{\perp}\right)\right)^{+}
\end{aligned}
$$

where $\lambda_{k}^{\perp}\left(k=1,2, \ldots, N_{i}^{t}-\sum_{j \neq i} N_{i}^{r}\right)$ are the eigenvalues of the user $i$ 's effective channel matrix after projection to

$$
\begin{aligned}
& \mathbf{H}_{i i}\left[\mathbf{I}-\mathbf{H}_{-i}^{H}\left(\mathbf{H}_{-i} \mathbf{H}_{-i}^{H}\right)^{-1} \mathbf{H}_{-i}\right] \\
& \cdot\left[\mathbf{I}-\mathbf{H}_{-i}^{H}\left(\mathbf{H}_{-i} \mathbf{H}_{-i}^{H}\right)^{-1} \mathbf{H}_{-i}\right]^{H} \mathbf{H}_{i i}^{H} .
\end{aligned}
$$

The second equality in (20) is obtained by interpreting the FP precoding as an optimal precoding of the effective channel matrix after null-space projection.

The rate of user $i$ employing FDM when using $t_{i} \in[0,1]$ fraction of the spectrum can be written as

$$
\begin{aligned}
R_{i}^{\mathrm{FDM}}\left(t_{i}\right) & =t_{i} \log _{2} \operatorname{det}\left(\mathbf{I}+\frac{\mathbf{H}_{i i} \mathbf{Q}_{\mathrm{MR}_{i}}\left(p_{i}\right) \mathbf{H}_{i i}^{H}}{t_{i} \mathbf{I}}\right) \\
& =t_{i} \sum_{k=1}^{N_{i}^{r}}\left(\log _{2}\left(p_{i} \frac{\lambda_{i}^{\mathrm{MR}}}{t_{i}}\right)\right)^{+} .
\end{aligned}
$$

Let $t_{i} \sum_{k=1}^{N_{i}^{r}}\left(\log _{2}\left(p_{i} \lambda_{i}^{\mathrm{MR}} / t_{i}\right)\right)^{+}=R_{i}^{\mathrm{NB} \mathrm{FP}}$. Then, we can use the following criteria to determine the optimality of $\mathrm{NB}_{\mathrm{FP}}$ and $\mathrm{NB}_{\mathrm{FDM}}$ solutions:

- when $\sum_{i=1}^{M} t_{i}>1$, the $\mathrm{NB}_{\mathrm{FP}}$ solution outperforms the FDMbased counterpart;

- when $\sum_{i=1}^{M} t_{i}<1$, the $\mathrm{NB}_{\mathrm{FDM}}$ solution outperforms the $\mathrm{NB}_{\mathrm{FP}}$ one.

\section{NUMERICAL StUdiES}

We investigate the impact of the INRs on the convexity of the rate region quantitatively via numerical studies. Consider a 2-user Rayleigh fading MIMO interference system with parameters $\sigma=1$ and $N_{i}^{t}=$ $N_{i}^{r}=2, i=1,2$. We examine the probability that the rate region is convex for different values of signal-to-interference ratio (SIR). It can be seen in Fig. 2 that when the SNR is $-10,0$, and $10 \mathrm{~dB}$, the SIR should be at least 10,15 , and $20 \mathrm{~dB}$, respectively, to ensure that the rate region is convex. Two trends can be observed in Fig. 2: i) as the SIR increases, i.e., as the INR decreases while the SNR is fixed, the probability that the rate region is convex increases; ii) as the SNR increases, the SIR needs to increase as well to retain the probability that the rate region is convex.

In Fig. 3, we evaluate the probability of the existence of the $\mathrm{NB}_{\mathrm{FP}}$ solution, i.e., the probability that the use of FP precoding is strictly optimal over competition among users in terms of resultant rates. As we can see from Fig. 3, the probability of the existence of $\mathrm{NB}_{\mathrm{FP}}$ solution increases with the increase of INR, which infers that the FP precoding becomes more favorable than the NE as the INR increases. This is because the rates of the $\mathrm{NB}_{\mathrm{FP}}$ solution do not change against INRs, but

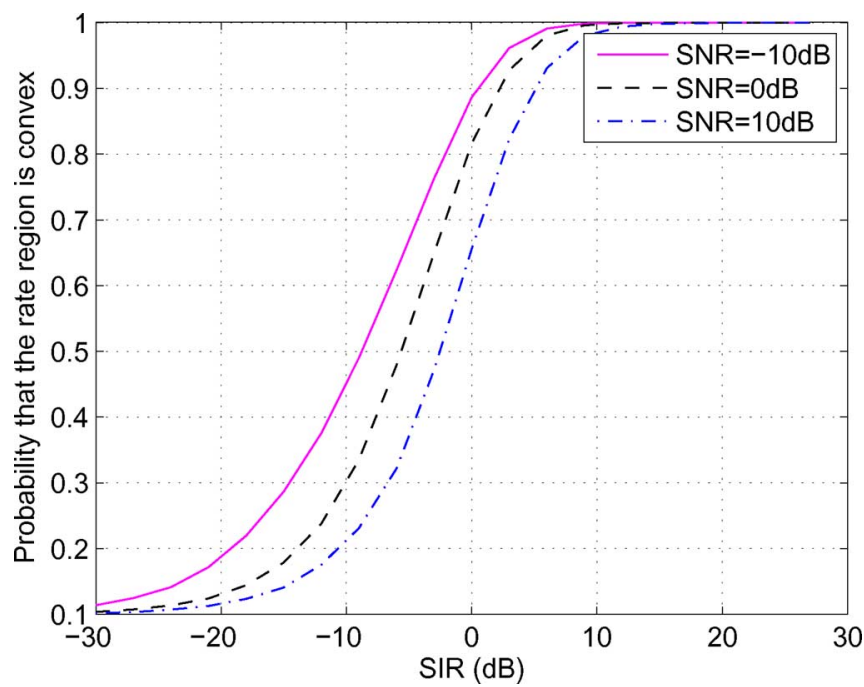

Fig. 2. Probability of convex rate region over different values of SIR.

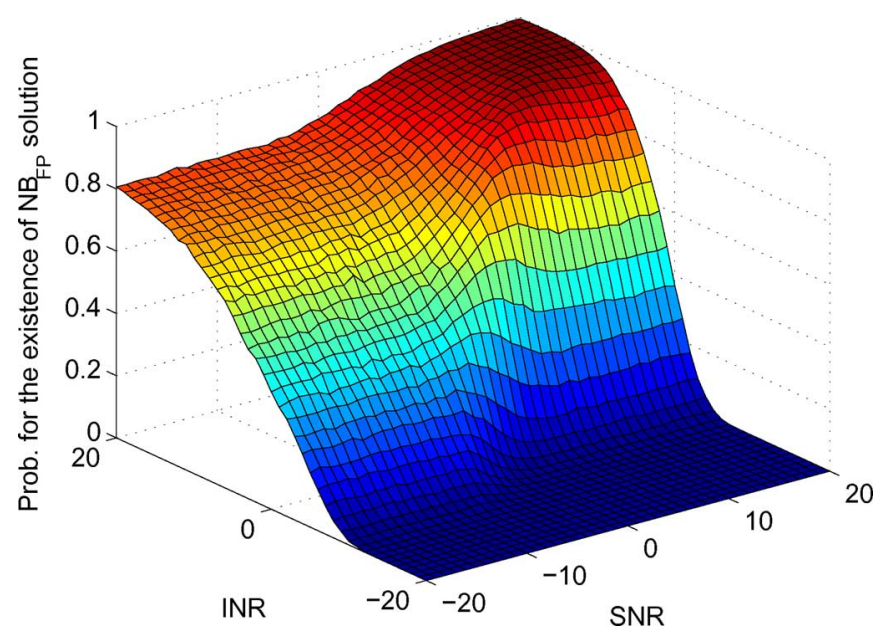

Fig. 3. Existence of the $\mathrm{NB}_{\mathrm{FP}}$ solution for different SNRs and INRs.

the rates of the NE decrease as the INR increases due to the rising interference. Another interesting phenomenon that can be observed from Fig. 3 is that the probability for the existence of the $\mathrm{NB}_{\mathrm{FP}}$ solution is not sensitive to SNR. However, at large INRs this probability increases slightly with the increase of SNR. This is due to the fact that given a transmission power for FP precoding the number of effective transmission streams tends to increase as the SNR increases. Meanwhile, the number of effective transmission streams almost does not change over SNRs for the precoding of NE, since at large INR the effective transmission streams of NE for each user are mainly determined by its interfering channel rather than the desired channel, i.e., the SNR has little impact on the number of effective transmission streams.

\section{CONCLUSION}

The rate control problem in multiuser MIMO interference systems has been formulated as an MCO problem. The convexity of the Pareto rate region of this $\mathrm{MCO}$ problem has been studied. A sufficient condition which guarantees the convexity of the rate region has been derived. Various rate region convexification approaches including the multistage interference cancellation and FP-based interference avoidance schemes have been analyzed for the MIMO interference system. It is argued that the interference mitigation techniques are preferable for 
convexifying the Pareto rate region over other existing techniques in terms of resultant user rates. Then, the MCO problem has been transformed into a single-objective optimization problem by using NB. A variety of characteristics for NB in MIMO interference systems such as the uniqueness and the optimality of different NB solutions have been considered. A sufficient condition ensuring the uniqueness of the purestrategy NB solution in MIMO interference systems has been derived. A method to determine the optimality among FP- and TDM-based NB solutions has been presented as well. Finally, the convexity of the rate region and the existence of the FP-based NB solution have also been demonstrated via numerical studies.

\section{REFERENCES}

[1] R. M. Beeson and W. S. Meisel, "The optimization of complex systems with respect to multiple criteria," in Proc. IEEE Syst., Man Cybern. Group Ann. Symp., Anaheim, CA, Oct. 1971, pp. 144-149.

[2] L. Zadeh, "Optimality and non-scalar-valued performance criteria," IEEE Trans. Autom. Control, vol. AC-8, no. 1, pp. 59-60, Jan. 1963.

[3] E. Larsson and E. Jorswieck, "Competition versus collaboration on the MISO interference channel," IEEE J. Sel. Areas Commun., vol. 26, no. 7, pp. 1059-1069, Sep. 2008.

[4] A. Leshem and E. Zehavi, "Bargaining over the interference channel," in Proc. IEEE ISIT, Seattle, WA, Jul. 2006, pp. 2225-2229.

[5] J. Gao, S. A. Vorobyov, and H. Jiang, "Cooperative resource allocation games under spectral mask and total power constraints," IEEE Trans. Signal Process., vol. 58, no. 8, pp. 4379-4395, Aug. 2010

[6] J. F. Nash, "The bargaining problem," Econometrica, vol. 18, no. 2 , pp. 155-162, Apr. 1950.

[7] A. Leshem and E. Zehavi, "Cooperative game theory and the Gaussian interference channel," IEEE J. Sel. Areas Commun., vol. 26, no. 7, pp. 1078-1088, Sep. 2008.

[8] M. Nokleby, A. L. Swindlehurst, R. Yue, and Y. Hua, "Cooperative power scheduling for wireless MIMO networks," in Proc. IEEE GLOBECOM'07, Washington, DC, Nov. 2007, pp. 2982-2986.

[9] G. Arslan, M. F. Demirkol, and Y. Song, "Equilibrium efficiency improvement in MIMO interference systems: A decentralized stream control approach," IEEE Trans. Wireless Commun., vol. 6, no. 8, pp. 2984-2993, Aug. 2007.

[10] F. R. Farrokhi, G. J. Foschini, A. Lozano, and R. A. Valenzuela, "Linkoptimal space-time processing with multiple transmit and receive antennas," IEEE Commun. Lett., vol. 5, no. 3, pp. 85-87, Mar. 2001.

[11] M. J. Osborne and A. Rubinstein, A Course in Game Theory. Cambridge, MA: MIT Press, 1994.

[12] W. Yu, W. Rhee, S. Boyd, and J. M. Cioffi, "Iterative water-filling for Gaussian vector multiple-access channels," IEEE Trans. Inf. Theory, vol. 50, no. 1, pp. 145-152, Jan. 2004.

[13] Z. Chen, S. A. Vorobyov, C.-X. Wang, and J. Thompson, "Pareto region characterization for rate control in multi-user systems and Nash bargaining," Tech. Rep., 2010 [Online]. Available: http://arxiv.org/abs/ 1006.1380

[14] X. Hong, Z. Chen, C.-X. Wang, S. A. Vorobyov, and J. Thompson, "Cognitive radio networks: Interference cancellation and management techniques," IEEE Veh. Technol. Mag., vol. 4, no. 4, pp. 76-84, Nov. 2009.

[15] Z. Chen, C.-X. Wang, X. Hong, J. Thompson, S. A. Vorobyov, F. Zhao, and X. Ge, "Interference mitigation for cognitive radio MIMO systems based on practical precoding,", Elsevier Phys. Commun. 2012 [Online]. Available: http://dx.doi.org/10.1016/j.phycom.2012.04.007

[16] E. Telatar, "Capacity of multiantenna Gaussian channels," AT\&T Bell Lab., Tech. Memo, 1995.

[17] S. Boyd and L. Vandenberghe, Convex Optimization. Cambridge, U.K.: Cambridge Univ. Press, 2004.

[18] G. Scutari, D. P. Palomar, and S. Barbarossa, "Optimal linear precoding strategies for wideband noncooperative systems based on game theory-Part I: Nash equilibria," IEEE Trans. Signal Process., vol. 56, no. 3, pp. 1230-1249, Mar. 2008.

\section{Worst-Case Mahler Measure in Polytopic Uncertain Systems}

\author{
Graziano Chesi, Senior Member, IEEE
}

\begin{abstract}
The Mahler measure provides a way to quantify the unstable and plays a key role in stabilization problems. This technical brief addresses the computation of the worst-case Mahler measure in systems depending polynomially on uncertain parameters constrained in a polytope. A sufficient condition for establishing an upper bound of the worst-case Mahler measure is provided in terms of linear matrix inequality (LMI) feasibility tests, where a homogeneous parameter-dependent quadratic Lyapunov function (HPD-QLF) is searched for. Moreover, it is shown that the best upper bound guaranteed by this condition can be obtained by solving generalized eigenvalue problems. Then, the conservatism of this methodology is investigated, showing that the upper bound is monotonically nonincreasing with the degree of the HPD-QLF, and that there exists a degree for which the upper bound is guaranteed to be tight. Some numerical examples illustrate the proposed results.
\end{abstract}

Index Terms-Linear matrix inequality (LMI), Mahler measure, networked control system, robustness, uncertainty.

\section{INTRODUCTION}

The Mahler measure [1], i.e., the absolute product of the unstable eigenvalues of a matrix, provides a way to quantify the unstable in discrete-time linear systems, see in particular the recent work [2]. This measure plays a key role in control systems. For instance, in networked control systems, an important issue is stabilization with information constraint in the input channel, see e.g., [3]-[6]. This information constraint can be modeled in several ways including data-rate constraint [7], [8], quantization [9], and signal-to-noise ratio [10]. As it has been shown in the literature, solutions for this issue can be obtained in terms of the Mahler measure of the system, see e.g., [11], [12].

Unfortunately, the model of a control system is not exactly known in general. In fact, its coefficients can be affected by uncertain parameters, for instance representing physical quantities that cannot be measured exactly or that are subject to changes. This means that analysis and control issues should consider not just one model but instead a family of admissible ones. In terms of the Mahler measure, hence, it appears important to determine the worst-case value among all the admissible models.

Systems with uncertainty can be modeled in various ways. One of the most used in the literature is known as polytopic description of the uncertainty and consists of expressing the coefficients of the system as functions of uncertain parameters constrained into a bounded convex polytope. This description includes the standard case of uncertain systems affected by scalar parameters constrained into intervals, and has been adopted for addressing numerous issues in systems with uncertainty, such as robust stability, robust performance, and robust control, see e.g., [13]-[17] and references therein among many contributions. Before proceeding it is worth mentioning that the uncertainty can be modeled also in other ways, e.g., through quadratic forms as done in [18].

Manuscript received November 21, 2011; revised February 21, 2012, April 10, 2012, and April 16, 2012; accepted April 23, 2012. Date of publication May 21, 2012; date of current version November 21, 2012. Recommended by Associate Editor T. Zhou.

The author is with the Department of Electrical and Electronic Engineering, University of Hong Kong, Hong Kong (e-mail: chesi@eee.hku.hk).

Digital Object Identifier 10.1109/TAC.2012.2199183 